\title{
Magnetic Dipole, Electric Quadrupole and Magnetic Octupole Moments of the $\Delta$ Baryons in Light Cone QCD Sum Rules
}

\author{
K. Azizi* \\ Physics Department, Middle East Technical University, 06531, Ankara, Turkey
}

\begin{abstract}
Due to the very short life time of the $\Delta$ baryons, a direct measurement on the electromagnetic moments of these systems is almost impossible in the experiment and can only be done indirectly. Although only for the magnetic dipole moments of $\Delta^{++}$and $\Delta^{+}$systems there are some experimental data, the theoretical, phenomenological and lattice calculations could play crucial role. In present work, the magnetic dipole $\left(\mu_{\Delta}\right)$, electric quadrupole $\left(Q_{\Delta}\right)$ and magnetic octupole $\left(O_{\Delta}\right)$ moments of these baryons are computed within the light cone QCD sum rules. The results are compared with the predictions of the other phenomenological approaches, lattice QCD and existing experimental data.
\end{abstract}

PACS: 11.55.Hx, 13.40.Em, 13.40.Gp, 14.20.-c

*e-mail:e146342@metu.edu.tr 


\section{Introduction}

Study of the electromagnetic properties of the baryons can give valuable information on their internal structure. Some of the main static electromagnetic parameters of the $\Delta$ baryons are their magnetic dipole $\left(\mu_{\Delta}\right)$, electric quadrupole $\left(Q_{\Delta}\right)$ and magnetic octupole $\left(O_{\Delta}\right)$ moments. The $\Delta^{-,+,++, 0}$ baryons are the lowest and very well-known nucleon resonances. Because of their too short mean life time $\left(\sim 10^{-23} \mathrm{~s}\right)$, there is almost no direct experimental information about their form factors and electromagnetic moments. An indirect measurement for the magnetic dipole moment of $\Delta^{++}$was accurately done from the radiative pion-nucleon scattering [1] (see [2] for experimental values of the magnetic dipole moment of $\Delta^{++}$obtained from various experiments). The magnetic moment of the $\Delta^{+}$resonance has also been measured via $\gamma P \rightarrow \pi^{0} \gamma^{\prime} P$ reaction in [3].

The magnetic dipole moments of these baryons have been studied in the framework of the various theoretical approaches. The radiative pion production on the nucleon $\left(\gamma N \rightarrow \pi N \gamma^{\prime}\right)$ with the aim of the determination of the magnetic dipole moment of the $\Delta^{+}(1232)$ has been studied in the frame work of Chiral effective- field theory in [4]. The magnetic dipole moment for $\Delta$ baryons is calculated in the framework of the static quark model (SQM) [5], relativistic quark models (RQM) [6], QCD sum rules (QCDSR) 7, 8], Chiral quark-soliton models (ChQSM) 9], heavy baryon Chiral perturbation theory (HBChPT) [10, 11], a phenomenological quark model (PQM) which nonstatic effects of pion exchange and orbital excitation are included [12], Lattice QCD [13, 14, 15, 16] and Chiral effective-field theory [17]. The magnetic dipole, electric quadrupole and magnetic octupole moments of these baryons is also calculated in [18] in the spectator quark formalism based on a simple $\Delta$ wave function corresponding to a quark-diquark system in an S-state. In [19], the 
Quadrupole Moment of the $\Delta$ baryons are calculated in the frame work of the consistituent quark model. Recently, the octupole moments of the light decuplet baryons are reported in [20] within the non-covariant quark model.

In the present work, we study the magnetic dipole, electric quadrupole and magnetic octupole moments of the $\Delta$ baryons in light cone QCD sum rules (LCSR) approach. Note that, by calculating the electromagnetic form factors, the electromagnetic dipole moments of the nucleons have been studied in [21] in the same frame work. The paper contains 3 sections. In section 2 , the light cone QCD sum rules for the magnetic dipole, electric quadrupole and magnetic octupole moments are calculated. Section 3 is devoted to the numerical analysis of the sum rules, a comparison of our results with the predictions of the other approaches as well as the existing experimental data and also discussion.

\section{Light cone QCD sum rules for the magnetic dipole, electric quadrupole and magnetic octupole moments of the $\Delta$ baryons}

To calculate the magnetic dipole, electric quadrupole and magnetic octupole moments of the $\Delta$ baryons, we start considering the basic object in LCSR method (the correlation function), where hadrons are represented by the interpolating quark currents.

$$
T_{\mu \nu}=i \int d^{4} x e^{i p x}\left\langle 0\left|T\left\{\eta_{\mu}(x) \bar{\eta}_{\nu}(0)\right\}\right| 0\right\rangle_{\gamma},
$$

where $\eta_{\mu}$ is the interpolating current of the $\Delta$ baryons and $\gamma$ stands for the electromagnetic field. In QCD sum rules approach, this correlation function is calculated in two different languages: in the quark-gluon language $(\mathrm{QCD}$ or theoretical side), it describes a hadron as quarks and gluons interacting 
in $\mathrm{QCD}$ vacuum. In the physical side, it is saturated by complete sets of hadrons with the same quantum numbers as their interpolating currents. The physical quantities, i.e., the electromagnetic form factors and multipole moments are calculated equating these two different representations of the correlation function.

The physical or phenomenological side of the correlation function can be obtained inserting the complete sets of the hadronic states between the interpolating currents in Eq. (1) with the same quantum numbers as the $\Delta$ baryons, i.e.,

$$
T_{\mu \nu}=\frac{\left\langle 0\left|\eta_{\mu}\right| \Delta\left(p_{2}\right)\right\rangle}{p_{2}^{2}-m_{\Delta}^{2}}\left\langle\Delta\left(p_{2}\right) \mid \Delta\left(p_{1}\right)\right\rangle_{\gamma} \frac{\left\langle\Delta\left(p_{1}\right)\left|\bar{\eta}_{\nu}\right| 0\right\rangle}{p_{1}^{2}-m_{\Delta}^{2}},
$$

where $p_{1}=p+q, p_{2}=p$ and $\mathrm{q}$ is the momentum of the photon. The matrix element of the interpolating current between the vacuum and the baryon state is defined as

$$
\left\langle 0\left|\eta_{\mu}(0)\right| \Delta(p, s)\right\rangle=\lambda_{\Delta} u_{\mu}(p, s),
$$

where $\lambda_{\Delta}$ is the residue and $u_{\mu}(p, s)$ is the Rarita-Schwinger spinor. The matrix element $\left\langle\Delta\left(p_{2}\right) \mid \Delta\left(p_{1}\right)\right\rangle_{\gamma}$ entering Eq. (2) can be parameterized in terms of some form factors as [18, 23, 24]:

$$
\begin{aligned}
\left\langle\Delta\left(p_{2}\right) \mid \Delta\left(p_{1}\right)\right\rangle_{\gamma} & =-e \bar{u}_{\mu}\left(p_{2}\right)\left\{F_{1} g^{\mu \nu} \notin-\frac{1}{2 m_{\Delta}}\left[F_{2} g^{\mu \nu}+F_{4} \frac{q^{\mu} q^{\nu}}{\left(2 m_{\Delta}\right)^{2}}\right] \notin \not q\right. \\
& \left.+F_{3} \frac{1}{\left(2 m_{\Delta}\right)^{2}} q^{\mu} q^{\nu} \notin\right\} u_{\nu}\left(p_{1}\right),
\end{aligned}
$$

where $\varepsilon$ is the polarization vector of the photon and $F_{i}$ are the form factors as functions of $q^{2}=\left(p_{1}-p_{2}\right)^{2}$. In obtaining the expression for the correlation function, summation over spins of the $\Delta$ particles is performed using

$$
\sum_{s} u_{\mu}(p, s) \bar{u}_{\nu}(p, s)=\frac{\left(\not p+m_{\Delta}\right)}{2 m_{\Delta}}\left\{-g_{\mu \nu}+\frac{1}{3} \gamma_{\mu} \gamma_{\nu}-\frac{2 p_{\mu} p_{\nu}}{3 m_{\Delta}^{2}}-\frac{p_{\mu} \gamma_{\nu}-p_{\nu} \gamma_{\mu}}{3 m_{\Delta}}\right\}
$$


In deriving the expression for the phenomenological side of the correlation function appear two problems (see also [22]): 1) all Lorentz structures are not independent, 2 ) not only spin $3 / 2$, but spin $1 / 2$ states also contribute to the correlator, which should be eliminated. Indeed, the matrix element of the current $\eta_{\mu}$ between vacuum and spin $1 / 2$ states is nonzero and is determined as

$$
\left\langle 0\left|\eta_{\mu}(0)\right| B(p, s=1 / 2)\right\rangle=\left(A p_{\mu}+B \gamma_{\mu}\right) u(p, s=1 / 2) .
$$

Imposing the condition $\gamma_{\mu} \eta^{\mu}=0$, one can immediately obtain that $B=$ $-\frac{A}{4} m$.

To remove the spin $1 / 2$ contribution and obtain only independent structures in the correlation function, we order Dirac matrices in a specific form. For this aim, we choose the ordering for Dirac matrices as $\gamma_{\mu} \not p \not \subset \not \gamma_{\nu}$. With this ordering for the correlator, we obtain

$$
\begin{aligned}
T_{\mu \nu} & =-\lambda_{\Delta}^{2} \frac{1}{\left(p_{1}^{2}-m_{\Delta}^{2}\right)\left(p_{2}^{2}-m_{\Delta}^{2}\right)}\left[2 m_{\Delta}(\varepsilon \cdot p) g_{\mu \nu} F_{1}\right. \\
& +\frac{1}{m_{\Delta}}(\varepsilon \cdot p) g_{\mu \nu} \not p \not q F_{2}+\frac{1}{2 m_{\Delta}^{2}}(\varepsilon \cdot p) q_{\mu} q_{\nu} \not p F_{3} \\
+ & \frac{1}{4 m_{\Delta}^{2}}(\varepsilon \cdot p) q_{\mu} q_{\nu} \not F_{4}+\text { other independent structures } \\
+ & \text { structures with } \gamma_{\mu} \text { at the beginning and } \gamma_{\nu} \text { at the end } \\
& \text { or which are proportional to } \left.p_{2 \mu} \text { or } p_{1 \nu}\right] .
\end{aligned}
$$

The magnetic dipole $\left(G_{m}\left(q^{2}\right)\right)$, electric quadrupole $\left(G_{Q}\left(q^{2}\right)\right)$ and magnetic octupole $\left(G_{O}\left(q^{2}\right)\right)$ form factors are defined in terms of the form factors $F_{i}\left(q^{2}\right)$ in the following way [18, 23, 24, 25]:

$$
\begin{aligned}
G_{m}\left(q^{2}\right) & =\left[F_{1}\left(q^{2}\right)+F_{2}\left(q^{2}\right)\right]\left(1+\frac{4}{5} \tau\right)-\frac{2}{5}\left[F_{3}\left(q^{2}\right)+F_{4}\left(q^{2}\right)\right] \tau(1+\tau) \\
G_{Q}\left(q^{2}\right) & =\left[F_{1}\left(q^{2}\right)-\tau F_{2}\left(q^{2}\right)\right]-\frac{1}{2}\left[F_{3}\left(q^{2}\right)-\tau F_{4}\left(q^{2}\right)\right](1+\tau) \\
G_{O}\left(q^{2}\right) & =\left[F_{1}\left(q^{2}\right)+F_{2}\left(q^{2}\right)\right]-\frac{1}{2}\left[F_{3}\left(q^{2}\right)+F_{4}\left(q^{2}\right)\right](1+\tau),
\end{aligned}
$$


where $\tau=-\frac{q^{2}}{4 m_{\Delta}^{2}}$. At $q^{2}=0$, the multipole form factors are obtained in terms of the functions $F_{i}(0)$ as:

$$
\begin{aligned}
G_{m}(0) & =F_{1}(0)+F_{2}(0) \\
G_{Q}(0) & =F_{1}(0)-\frac{1}{2} F_{3}(0) \\
G_{O}(0) & =F_{1}(0)+F_{2}(0)-\frac{1}{2}\left[F_{3}(0)+F_{4}(0)\right] .
\end{aligned}
$$

The static magnetic dipole $\left(\mu_{\Delta}\right)$, electric quadrupole $\left(Q_{\Delta}\right)$ and magnetic octupole $\left(O_{\Delta}\right)$ moments in their natural magneton are defined in the following way:

$$
\begin{aligned}
\mu_{\Delta} & =\frac{e}{2 m_{\Delta}} G_{m}(0) \\
Q_{\Delta} & =\frac{e}{m_{\Delta}^{2}} G_{Q}(0) \\
O_{\Delta} & =\frac{e}{2 m_{\Delta}^{3}} G_{O}(0)
\end{aligned}
$$

The theoretical part of the correlation function can be calculated in light cone QCD sum rules via the operator product expansion (OPE) in deep Euclidean region where $p^{2} \ll 0$ and $(p+q)^{2} \ll 0$ in terms of the photon distribution amplitudes (DA's). To calculate the correlation function from theoretical or QCD side, the explicit expressions of the interpolating currents of the $\Delta$ baryons are needed. The interpolating current for $\Delta^{+}$is chosen as

$$
\eta_{\mu}=\frac{1}{\sqrt{3}} \varepsilon^{a b c}\left[2\left(u^{a T} C \gamma_{\mu} d^{b}\right) u^{c}+\left(u^{a T} C \gamma_{\mu} u^{b}\right) d^{c}\right],
$$

where $\mathrm{C}$ is the charge conjugation operator and $\mathrm{a}, \mathrm{b}$ and $\mathrm{c}$ are color indices. Here we should mention that in the present work, first we calculate the correlation function for $\Delta^{+}$then with the help of the relations which we will present next, the correlators of $\Delta^{-}, \Delta^{++}$and $\Delta^{0}$ will be obtained using the correlation function of the $\Delta^{+}$. After contracting out the quark pairs in Eq. 
(1) by the help of the Wick's theorem, we obtain the following expression for the correlation function in terms of the quark propagators

$$
\begin{aligned}
\Pi_{\mu \nu} & =\frac{i}{3} \epsilon_{a b c} \epsilon_{a^{\prime} b^{\prime} c^{\prime}} \int d^{4} x e^{i p x}\langle 0[\gamma(q)]|\left\{2 S_{d}^{c a^{\prime}} \gamma_{\nu} S_{u}^{\prime a b^{\prime}} \gamma_{\mu} S_{u}^{b c^{\prime}}\right. \\
& -2 S_{d}^{c a^{\prime}} \gamma_{\nu} S_{u}^{\prime b b^{\prime}} \gamma_{\mu} S_{u}^{a c^{\prime}}+2 S_{u}^{c a^{\prime}} \gamma_{\nu} S_{u}^{\prime a b^{\prime}} \gamma_{\mu} S_{d}^{b c^{\prime}}-2 S_{u}^{c b^{\prime}} \gamma_{\nu} S_{u}^{\prime a a^{\prime}} \gamma_{\mu} S_{d}^{b c^{\prime}} \\
& +4 S_{u}^{c b^{\prime}} \gamma_{\nu} S_{d}^{\prime b a^{\prime}} \gamma_{\mu} S_{u}^{a c^{\prime}}+\operatorname{Tr}\left(\gamma_{\mu} S_{u}^{a a^{\prime}} \gamma_{\nu} S_{u}^{\prime b b^{\prime}}\right) S_{d}^{c c^{\prime}} \\
& \left.-\operatorname{Tr}\left(\gamma_{\mu} S_{u}^{a b^{\prime}} \gamma_{\nu} S_{u}^{\prime b a^{\prime}}\right) S_{d}^{c c^{\prime}}-4 \operatorname{Tr}\left(\gamma_{\mu} S_{u}^{a b^{\prime}} \gamma_{\nu} S_{d}^{\prime b a^{\prime}}\right) S_{u}^{c c^{\prime}}\right\}|0\rangle,
\end{aligned}
$$

where $S^{\prime}=C S^{T} C$ and $S_{u, d}$ are the full light quark propagators, which their explicit expressions can be found in [26, 27] (see also [22, 28]). To calculate the above correlation function, we follow the same procedure as stated in [22, 28] and use the photon distribution amplitudes (DA's) calculated in [29]. For convenience, we present those DA's in the appendix-A.

Using the expressions of the full light propagator and the photon DA's and separating the coefficient of the structures $(\varepsilon \cdot p) g_{\mu \nu},(\varepsilon \cdot p) g_{\mu \nu} \not p \not q,(\varepsilon \cdot p) q_{\mu} q_{\nu} \not p$ and $(\varepsilon . p) q_{\mu} q_{\nu} \not q$ for the $F_{1}, F_{2}, F_{3}$ and $F_{4}$, respectively, the expressions of the correlation function from the QCD side are obtained. Separating the coefficient of the same structures from phenomenological part and equating these representations of the correlator, sum rules for the $F_{i}$ functions are obtained. In order to suppress the contribution of the higher states and continuum, Borel transformation with respect to the variables $p_{2}^{2}=p^{2}$ and $p_{1}^{2}=(p+q)^{2}$ is applied. The explicit expressions for $F_{i}$ are given in the appendix-B.

At the end of this section, we would like to present some relations between the correlation functions. Our calculations show that the coefficient of any structure in the correlation function of the $\Delta^{+}$can be written in the form

$$
\Pi^{\Delta^{+}}=-\frac{1}{6}\left(2 e_{u}+e_{d}\right) \mathcal{H}(u, d),
$$


where the function $\mathcal{H}(u, d)$ depends on the masses and condensates of the $\mathrm{u}$ and $\mathrm{d}$ quarks and it is independent of the charge of the quarks. Our calculations indicate that the $\Pi^{\Delta^{-, 0,++}}$ can be obtained from the $\Pi^{\Delta^{+}}$by the following replacements:

$$
\begin{aligned}
\Pi^{\Delta^{++}} & =\Pi^{\Delta^{+}}(d \rightarrow u)=-\frac{1}{2} e_{u} \mathcal{H}(u, u), \\
\Pi^{\Delta^{0}} & =\Pi^{\Delta^{+}}(d \leftrightarrow u)=-\frac{1}{6}\left(2 e_{d}+e_{u}\right) \mathcal{H}(d, u), \\
\Pi^{\Delta^{-}} & =\Pi^{\Delta^{+}}(u \rightarrow d)=-\frac{1}{2} e_{d} \mathcal{H}(d, d),
\end{aligned}
$$

We consider the massless quarks, $m_{u}=m_{d}=0$, and exact $\mathrm{SU}(2)$ flavor symmetry implying $\langle\bar{u} u\rangle=\langle\bar{d} d\rangle$. Under exact $S U(2)$ flavor symmetry, $\mathcal{H}(u, d)=\mathcal{H}(d, u)=\mathcal{H}(u, u)=\mathcal{H}(d, d)=\mathcal{H}$ and the following relations are obtained (see also [8]):

$$
\begin{aligned}
\Pi^{\Delta^{++}} & =-\frac{1}{2} e_{u} \mathcal{H}, \\
\Pi^{\Delta^{+}} & =-\frac{1}{6}\left(2 e_{u}+e_{d}\right) \mathcal{H}, \\
\Pi^{\Delta^{0}} & =-\frac{1}{6}\left(2 e_{d}+e_{u}\right) \mathcal{H}, \\
\Pi^{\Delta^{-}} & =-\frac{1}{2} e_{d} \mathcal{H},
\end{aligned}
$$

From above equation, by substituting the charge of the $\mathrm{u}$ and $\mathrm{d}$ quarks, the following exact relations between theoretical parts of the correlator of $\Delta$ baryons are derived:

$$
\begin{aligned}
\Pi^{\Delta^{+}} & =-\Pi^{\Delta^{-}}=\frac{1}{2} \Pi^{\Delta^{++}} \\
\Pi^{\Delta^{0}} & =0
\end{aligned}
$$




\section{$3 \quad$ Numerical analysis}

Present section encompasses the numerical analysis for the, magnetic dipole, electric quadrupole and magnetic octupole moments of the $\Delta$ baryons. The values for input parameters used in the analysis of the sum rules for the $F_{1}, F_{2}, F_{3}$ and $F_{4}$ are : $\langle\bar{u} u\rangle(1 \mathrm{GeV})=\langle\bar{d} d\rangle(1 \mathrm{GeV})=-(0.243)^{3} \mathrm{GeV}^{3}$, $ß(1 \mathrm{GeV})=0.8\langle\bar{u} u\rangle(1 \mathrm{GeV}), m_{0}^{2}(1 \mathrm{GeV})=(0.8 \pm 0.2) \mathrm{GeV}^{2}$ [30] and $f_{3 \gamma}=$ $-0.0039 \mathrm{GeV}^{2}[29]$. The value of the magnetic susceptibility was obtained in various papers as $\chi(1 \mathrm{GeV})=-3.15 \pm 0.3 \mathrm{GeV}^{-2}$ [29], $\chi(1 \mathrm{GeV})=$ $-(2.85 \pm 0.5) \mathrm{GeV}^{-2}$ [31] and $\chi(1 \mathrm{GeV})=-4.4 \mathrm{GeV}^{-2}[32$. The residue $\lambda_{\Delta}$ determined from mass sum rules and is taken to be $\lambda_{\Delta}=0.038 \mathrm{GeV}^{3}$ [7, 33, 34]. From sum rules for the $F_{1}, F_{2}, F_{3}$ and $F_{4}$, it follows that the photon DA's are also needed [29]. Their explicit expressions are also given in the appendix-A.

The sum rules for the magnetic dipole, electric quadrupole and magnetic octupole moments also contain two auxiliary parameters: Borel mass parameter $M^{2}$ and continuum threshold $s_{0}$. The physical quantities, i.e., magnetic dipole, electric quadrupole and magnetic octupole moments, should be independent of these parameters. The working region for $M^{2}$ is determined requiring that the contributions of the higher states and continuum are effectively suppressed. This condition is satisfied in the region $1 \mathrm{GeV}^{2} \leq M^{2} \leq 1.5 \mathrm{GeV}^{2}$.

The dependency of the magnetic dipole moment $\mu_{\Delta}$, electric quadrupole $Q_{\Delta}$ and magnetic octupole $O_{\Delta}$ moments on Borel parameter $M^{2}$ are presented in Figs. 1-3 at fixed value of the continuum threshold $s_{0}=4 \mathrm{GeV}^{2}$. The magnetic dipole moment is presented in its natural magneton $\left(e \hbar / 2 m_{\Delta} c\right)$ while the electric quadrupole $\left(Q_{\Delta}\right)$ and magnetic octupole $\left(O_{\Delta}\right)$ moments are shown in $\mathrm{fm}^{2}$ and $\mathrm{fm}^{3}$, respectively. The conversion coefficient from the nat- 


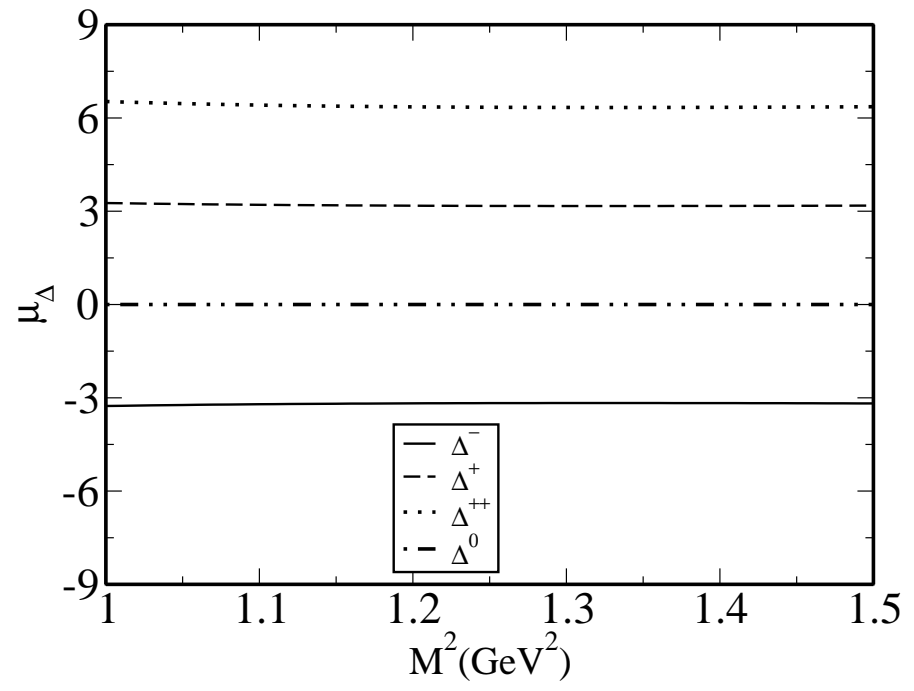

Figure 1: The dependence of the magnetic dipole moment $\mu_{\Delta}$ in its natural magneton on the Borel parameter $M^{2}$ at fixed value of the continuum threshold $s_{0}$

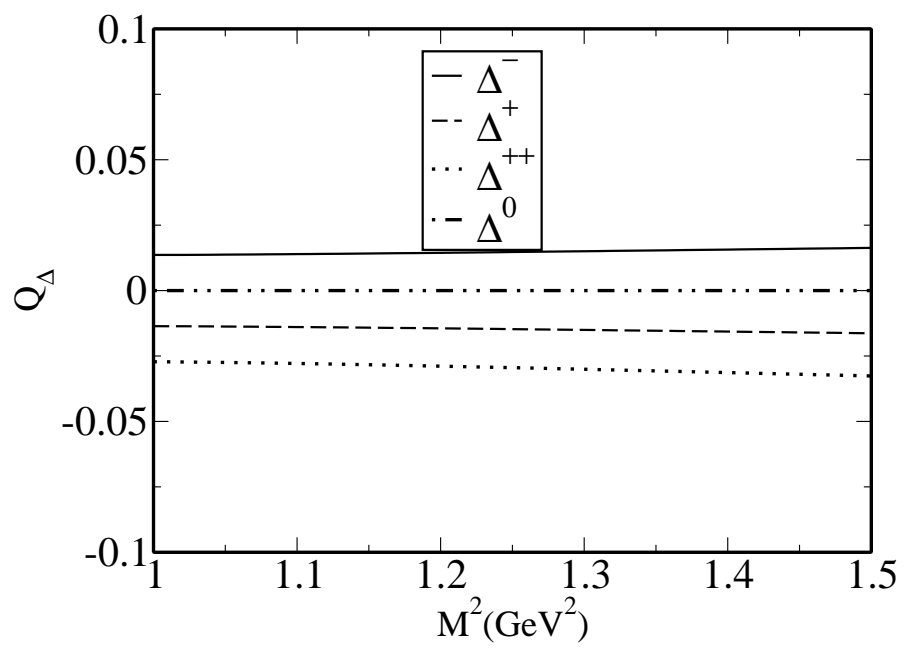

Figure 2: The dependence of the electric quadrupole $Q_{\Delta}$ in $\mathrm{fm}^{2}$ on the Borel parameter $M^{2}$ at fixed value of the continuum threshold $s_{0}=4 \mathrm{GeV}^{2}$. 


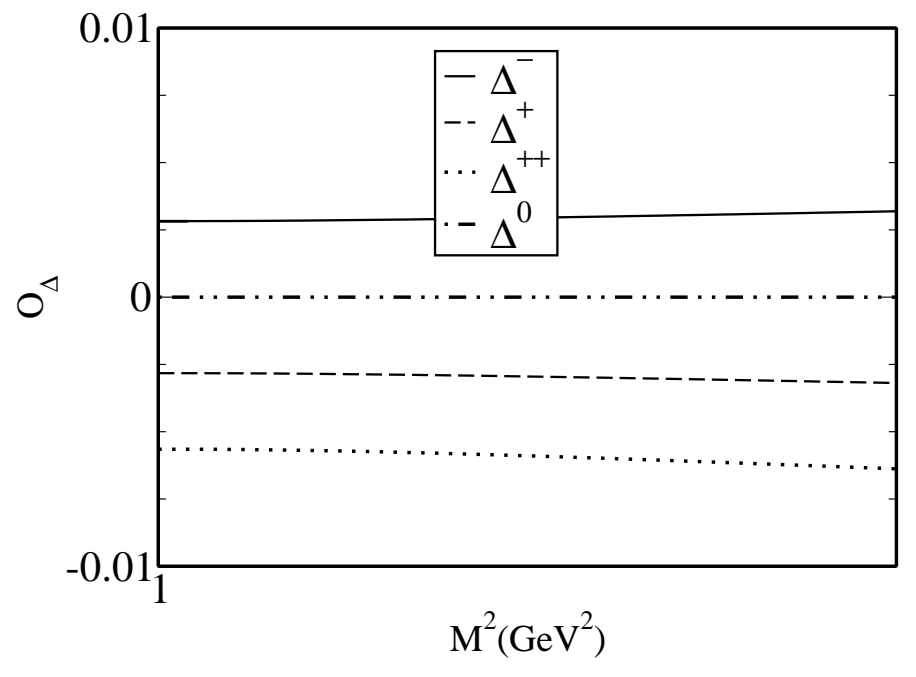

Figure 3: The same as Fig. 4, but for the magnetic octupole $O_{\Delta}$ in $\mathrm{fm}^{3}$.

ural magneton unit to the nucleon magneton is $\frac{m_{N}}{m_{\Delta}}$. Note that, our results are practically the same in the interval $s_{0}=(3.8-4.2) \mathrm{GeV}^{2}$ for continuum threshold. These figures present a good stability with respect to the Borel mass parameter. We should also mention that our results practically don't change considering three values of the $\chi$ as presented at the beginning of this section.

Our final results on the magnetic dipole moment $\mu_{\Delta}$ for $\Delta$ baryons are presented in Table 1. The quoted errors for the values are due to the uncertainties in the determination of the input parameters, the variation of $M^{2}$ as well as the systematic errors in QCD sum rules approach. For comparison, the predictions of other theoretical approaches, lattice QCD as well as the experiment are also presented. From this Table, we see a good consistency among the various approaches especially when we consider the errors except the lattice QCD prediction [15] for magnetic moment of $\Delta^{+}$.

We also depict the results of the electric quadrupole $Q_{\Delta}$ and magnetic 


\begin{tabular}{|c||c|c|c|c|}
\hline & $\Delta^{-}$ & $\Delta^{+}$ & $\Delta^{++}$ & $\Delta^{0}$ \\
\hline \hline present work & $-3.17 \pm 0.85$ & $3.17 \pm 0.85$ & $6.34 \pm 1.70$ & 0 \\
\hline Exp.[3] & - & $3.54_{-1.7}^{+1.3} \pm 1.96 \pm 3.93$ & - & - \\
\hline Exp.[2] & - & - & $7.34 \pm 2.49$ & - \\
\hline SQM[5] & -3.65 & 3.65 & 7.31 & 0 \\
\hline RQM[6] & -3.12 & 3.12 & 6.24 & 0 \\
\hline QCDSR[7] & $-2.88 \pm 0.52$ & $2.88 \pm 0.52$ & $5.76 \pm 1.05$ & 0 \\
\hline QCDSR[8] & $-2.71 \pm 0.85$ & $2.71 \pm 0.85$ & $5.41 \pm 1.70$ & 0 \\
\hline ChQSM[9] & -3.69 & 3.48 & 7.06 & -0.10 \\
\hline HBChPT[10] & $-2.95 \pm 0.33$ & $2.75 \pm 0.26$ & $6.24 \pm 0.52$ & $-0.22 \pm 0.05$ \\
\hline PQM[12] & - & 3.72 & 8.10 & - \\
\hline Lattice[13] & $-3.22 \pm 0.41$ & $3.22 \pm 0.41$ & $6.43 \pm 0.80$ & 0 \\
\hline Lattice[14] & $-3.22 \pm 0.35$ & $3.26 \pm 0.35$ & $6.54 \pm 0.73$ & 0.079 \\
\hline Lattice[15] & $-3.90 \pm 0.25$ & $1.27 \pm 0.10$ & $6.86 \pm 0.24$ & $-0.046 \pm 0.003$ \\
\hline Lattice[16] & - & $3.04 \pm 0.21$ & - & - \\
\hline Spectator[18] & -3.54 & 3.29 & 6.71 & -0.12 \\
\hline
\end{tabular}

Table 1: Comparison of the magnetic dipole moment $\mu_{\Delta}$ in units of its natural magneton for different approaches like static quark model (SQM) [5], relativistic quark models (RQM) [6], QCD sum rules (QCDSR) [7, 8], Chiral quark-soliton models (ChQSM) 9], heavy baryon Chiral perturbation theory (HBChPT) [10, a phenomenological quark model (PQM) which nonstatic effects of pion exchange and orbital excitation are included [12], Lattice QCD [13, 14, 15, 16] and experiment [2, 3]. The presented experimental value for $\Delta^{++}$is the average of sum data from [2].

octupole $O_{\Delta}$ moments in Table 2. In comparison, the results of the other approaches are also presented. From this Table, we see that the values for the electric quadrupole and magnetic octupole moments are very small in comparison with the magnetic dipole moment. Our results on electric quadrupole moments are consistent with the predictions of the constituent quark model with configuration mixing but no exchange currents (impulse approximation) [19] in order of magnitude, but about one order of magni- 


\begin{tabular}{|c||c|c|c|c|}
\hline & $\Delta^{-}$ & $\Delta^{+}$ & $\Delta^{++}$ & $\Delta^{0}$ \\
\hline \hline$Q_{\Delta}$ (present work) & $0.014 \pm 0.004$ & $-0.014 \pm 0.004$ & $-0.028 \pm 0.008$ & 0 \\
\hline$Q_{\Delta}[18]$ & 0 & 0 & 0 & 0 \\
\hline$Q_{\Delta}^{2 m p}[19]$ & 0.032 & -0.032 & -0.064 & 0 \\
\hline$Q_{\Delta}^{e x c}[19]$ & 0.119 & -0.119 & -0.238 & 0 \\
\hline \hline$O_{\Delta}$ (present work) & $0.003 \pm 0.001$ & $-0.003 \pm 0.001$ & $-0.006 \pm 0.002$ & 0 \\
\hline$O_{\Delta}[18]$ & 0 & 0 & 0 & 0 \\
\hline$O_{\Delta}[20]$ & 0.012 & -0.012 & -0.024 & 0 \\
\hline
\end{tabular}

Table 2: Results for the electric quadrupole $Q_{\Delta}$ in $f m^{2}$ and magnetic octupole $O_{\Delta}$ moments in $\mathrm{fm}^{3}$ in different approaches: LCSR(present work), spectator quark model([18]), constituent quark model with configuration mixing but no exchange currents (impulse approximation), constituent quark model with exchange currents but no configuration mixing [19] and noncovariant quark model [20].

tude smaller than the predictions of constituent quark model with exchange currents but no configuration mixing [19]. The [18] predicts no electric quadrupole and magnetic octupole moments for $\Delta$ baryons. Our results on the magnetic octupole moments for these baryons are about four times smaller than the predictions of the non-covariant quark model [20]. The negative sign in the value of the quadrupole and octupole moments of $\Delta^{+}$shows that the quadrupole and octupole distributions are oblate and have the same geometric shape as the charge distribution.

In conclusion, due to the very short life time, a direct measurement on the electromagnetic moments of $\Delta$ systems is almost not possible in the experiment and can only be done indirectly in a three-step process, where they are created, emit a low-energy photon and then decay. Although only for $\Delta^{+}$and $\Delta^{++}$systems there are some data, the theoretical, phenomenological and lattice calculations could play very important role. In present work, we 
computed the magnetic dipole, electric quadrupole and magnetic octupole moments of these baryons in the framework of the light cone QCD sum rules and compared their results with the predictions of the other phenomenological models, lattice QCD as well as the existing experimental data. The results depict that the electric quadrupole and magnetic octupole moments are very small in comparison with the magnetic dipole moment of these baryons.

\section{Acknowledgment}

The author would like to thank T. M. Aliev and A. Ozpineci for their useful discussions and also TUBITAK, Turkish Scientific and Research Council, for their partial financial support.

\section{References}

[1] B. M. K. Nefkens et al., Phys. Rev. D 18, 3911 (1978); A. Bosshard et al., Phys. Rev. D 44, 1962 (1991).

[2] W. M. Yao et al., Particle Data Group, J. Phys. G 33 (2006) 1.

[3] M. Kotulla et al., Phys. Rev. Lett. 89, 272001 (2002).

[4] V. Pascalutsa, M. Vanderhaeghen, Phys. Rev. D77, 014027 (2008).

[5] W. T. Chiang, M. Vanderhaeghen, S. N. Yang, D. Drechsel, Phys. Rev. C 71, 015204 (2005).

[6] F. Schlumpf, Phys. Rev. D 48, 4478 (1993).

[7] F. X. Lee, Phys. Rev. D 57, 1801 (1998); F. X. Lee, Phys. Lett. B 419, 14 (1998). 
[8] T. M. Aliev, A. Ozpineci, M. Savci, Nucl. Phys. A 678, 443 (2000).

[9] H. C. Kim, M. Praszalowicz, Phys. Lett. B 585, 99 (2004); H. C. Kim, M. Prasza- lowicz, K. Goeke, Phys. Rev. D 57, 2859 (1998).

[10] M. N. Butler, M. J. Savage, R. P. Springer, Phys. Rev. D 49, 3459 (1994).

[11] C. Hacker, N. Wies, J. Gegelia, S. Scherer, Eur. Phys. J. A 28, 5 (2006).

[12] J. Franklin, Phys. Rev. D 66, 033010 (2002).

[13] D. B. Leinweber, T. Draper, R. M. Woloshyn, Phys. Rev. D 46, 3067 (1992).

[14] I. C. Cloet, D. B. Leinweber, A. W. Thomas, Phys. Lett. B 563, 157 (2003); I. C. Cloet, D. B. Leinweber, A. W. Thomas, arXiv:nucl-th/0211027.

[15] F. X. Lee, R. Kelly, L. Zhou, W. Wilcox, Phys. Lett. B 627, 71 (2005).

[16] C. Alexandrou, T. Korzec, T. Leontiou, J. W. Negele, A. Tsapalis, PoS LATTICE2007, 149 (2006), arXiv:0710.2744 [hep-lat].

[17] V. Pascalutsa, M. Vanderhaeghen, Phys. Rev. Lett. 94, 102003 (2005).

[18] G. Ramalho, M.T. Pena, arXiv:0807.2922v1 [hep-ph].

[19] A. J. Buchmann arXiv:hep-ph/9909385v1, submitted to World Scientific on February 1, (2008).

[20] A. J. Buchmann, E. M. Henle, Eur. Phys. J. A 35, 267 (2008).

[21] T. M. Aliev, K. Azizi, A. Ozpineci, M. Savci, Phys. Rev. D 77, 114014 (2008). 
[22] T. M. Aliev, K. Azizi, A. Ozpineci, Nucl. Phys. B 808, 137 (2009), arXiv:0807.3481 [hep-ph].

[23] V. Pascalutsa, M. Vanderhaeghen, S. N. Yang, Phys. Rept. 437, 125 (2007) arXiv:hep-ph/0609004|.

[24] S. Nozawa, D. B. Leinweber, Phys. Rev. D 42, 3567 (1990).

[25] H. J. Weber, H. Arenhovel, Phys. Rept. 36 (1978) 277.

[26] I. I. Balitsky, V. M. Braun, Nucl. Phys. B 311, 541 (1989).

[27] V. M. Braun, I. E. Filyanov, Z. Phys. C 48, 239 (1990).

[28] T. M. Aliev, K. Azizi, A. Ozpineci, Phys. Rev. D 77, 114006 (2008).

[29] P. Ball, V. M. Braun, N. Kivel, Nucl. Phys. B 649, 263 (2003) .

[30] V. M. Belyaev, B. L. Ioffe, JETP 56, 493 (1982) .

[31] J. Rohrwild, JHEP 0709, 073 (2007).

[32] V. M. Belyaev, I. I. Kogan, Yad. Fiz. 40, 1035 (1984).

[33] W. Y. P. Hwang, K. C. Yang, Phys. Rev. D 49, 460 (1994).

[34] T. M. Aliev, K. Azizi, A. Ozpineci, Nucl. Phys. A 799, 105 (2008). 


\section{Appendix A}

The matrix elements used in the calculations are given in terms of the photon distribution amplitudes (DA's) as follows [29]:

$$
\begin{aligned}
& \left\langle\gamma(q)\left|\bar{q}(x) \sigma_{\mu \nu} q(0)\right| 0\right\rangle=-i e_{q} \bar{q} q\left(\varepsilon_{\mu} q_{\nu}-\varepsilon_{\nu} q_{\mu}\right) \int_{0}^{1} d u e^{i \bar{u} q x}\left(\chi \varphi_{\gamma}(u)+\frac{x^{2}}{16} \mathbb{A}(u)\right) \\
& -\frac{i}{2(q x)} e_{q}\langle\bar{q} q\rangle\left[x_{\nu}\left(\varepsilon_{\mu}-q_{\mu} \frac{\varepsilon x}{q x}\right)-x_{\mu}\left(\varepsilon_{\nu}-q_{\nu} \frac{\varepsilon x}{q x}\right)\right] \int_{0}^{1} d u e^{i \bar{u} q x} h_{\gamma}(u) \\
& \left\langle\gamma(q)\left|\bar{q}(x) \gamma_{\mu} q(0)\right| 0\right\rangle=e_{q} f_{3 \gamma}\left(\varepsilon_{\mu}-q_{\mu} \frac{\varepsilon x}{q x}\right) \int_{0}^{1} d u e^{i \bar{u} q x} \psi^{v}(u) \\
& \left\langle\gamma(q)\left|\bar{q}(x) \gamma_{\mu} \gamma_{5} q(0)\right| 0\right\rangle=-\frac{1}{4} e_{q} f_{3 \gamma} \epsilon_{\mu \nu \alpha \beta} \varepsilon^{\nu} q^{\alpha} x^{\beta} \int_{0}^{1} d u e^{i \bar{u} q x} \psi^{a}(u) \\
& \left\langle\gamma(q)\left|\bar{q}(x) g_{s} G_{\mu \nu}(v x) q(0)\right| 0\right\rangle=-i e_{q}\langle\bar{q} q\rangle\left(\varepsilon_{\mu} q_{\nu}-\varepsilon_{\nu} q_{\mu}\right) \int \mathcal{D} \alpha_{i} e^{i\left(\alpha_{\bar{q}}+v \alpha_{g}\right) q x} \mathcal{S}\left(\alpha_{i}\right) \\
& \left\langle\gamma(q)\left|\bar{q}(x) g_{s} \tilde{G}_{\mu \nu} i \gamma_{5}(v x) q(0)\right| 0\right\rangle=-i e_{q}\langle\bar{q} q\rangle\left(\varepsilon_{\mu} q_{\nu}-\varepsilon_{\nu} q_{\mu}\right) \int \mathcal{D} \alpha_{i} e^{i\left(\alpha_{\bar{q}}+v \alpha_{g}\right) q x} \tilde{\mathcal{S}}\left(\alpha_{i}\right) \\
& \left\langle\gamma(q)\left|\bar{q}(x) g_{s} \tilde{G}_{\mu \nu}(v x) \gamma_{\alpha} \gamma_{5} q(0)\right| 0\right\rangle=e_{q} f_{3 \gamma} q_{\alpha}\left(\varepsilon_{\mu} q_{\nu}-\varepsilon_{\nu} q_{\mu}\right) \int \mathcal{D} \alpha_{i} e^{i\left(\alpha_{\bar{q}}+v \alpha_{g}\right) q x} \mathcal{A}\left(\alpha_{i}\right) \\
& \left\langle\gamma(q)\left|\bar{q}(x) g_{s} G_{\mu \nu}(v x) i \gamma_{\alpha} q(0)\right| 0\right\rangle=e_{q} f_{3 \gamma} q_{\alpha}\left(\varepsilon_{\mu} q_{\nu}-\varepsilon_{\nu} q_{\mu}\right) \int \mathcal{D} \alpha_{i} e^{i\left(\alpha_{\bar{q}}+v \alpha_{g}\right) q x} \mathcal{V}\left(\alpha_{i}\right) \\
& \left\langle\gamma(q)\left|\bar{q}(x) \sigma_{\alpha \beta} g_{s} G_{\mu \nu}(v x) q(0)\right| 0\right\rangle=e_{q}\langle\bar{q} q\rangle\left\{\left[\left(\varepsilon_{\mu}-q_{\mu} \frac{\varepsilon x}{q x}\right)\left(g_{\alpha \nu}-\frac{1}{q x}\left(q_{\alpha} x_{\nu}+q_{\nu} x_{\alpha}\right)\right) q_{\beta}\right.\right. \\
& -\left(\varepsilon_{\mu}-q_{\mu} \frac{\varepsilon x}{q x}\right)\left(g_{\beta \nu}-\frac{1}{q x}\left(q_{\beta} x_{\nu}+q_{\nu} x_{\beta}\right)\right) q_{\alpha} \\
& -\left(\varepsilon_{\nu}-q_{\nu} \frac{\varepsilon x}{q x}\right)\left(g_{\alpha \mu}-\frac{1}{q x}\left(q_{\alpha} x_{\mu}+q_{\mu} x_{\alpha}\right)\right) q_{\beta} \\
& \left.+\left(\varepsilon_{\nu}-q_{\nu} \frac{\varepsilon x}{q \cdot x}\right)\left(g_{\beta \mu}-\frac{1}{q x}\left(q_{\beta} x_{\mu}+q_{\mu} x_{\beta}\right)\right) q_{\alpha}\right] \int \mathcal{D} \alpha_{i} e^{i\left(\alpha_{\bar{q}}+v \alpha_{g}\right) q x} \mathcal{T}_{1}\left(\alpha_{i}\right) \\
& +\left[\left(\varepsilon_{\alpha}-q_{\alpha} \frac{\varepsilon x}{q x}\right)\left(g_{\mu \beta}-\frac{1}{q x}\left(q_{\mu} x_{\beta}+q_{\beta} x_{\mu}\right)\right) q_{\nu}\right. \\
& -\left(\varepsilon_{\alpha}-q_{\alpha} \frac{\varepsilon x}{q x}\right)\left(g_{\nu \beta}-\frac{1}{q x}\left(q_{\nu} x_{\beta}+q_{\beta} x_{\nu}\right)\right) q_{\mu}
\end{aligned}
$$




$$
\begin{aligned}
& -\left(\varepsilon_{\beta}-q_{\beta} \frac{\varepsilon x}{q x}\right)\left(g_{\mu \alpha}-\frac{1}{q x}\left(q_{\mu} x_{\alpha}+q_{\alpha} x_{\mu}\right)\right) q_{\nu} \\
& \left.+\left(\varepsilon_{\beta}-q_{\beta} \frac{\varepsilon x}{q x}\right)\left(g_{\nu \alpha}-\frac{1}{q x}\left(q_{\nu} x_{\alpha}+q_{\alpha} x_{\nu}\right)\right) q_{\mu}\right] \int \mathcal{D} \alpha_{i} e^{i\left(\alpha_{\bar{q}}+v \alpha_{g}\right) q x} \mathcal{T}_{2}\left(\alpha_{i}\right) \\
& +\frac{1}{q x}\left(q_{\mu} x_{\nu}-q_{\nu} x_{\mu}\right)\left(\varepsilon_{\alpha} q_{\beta}-\varepsilon_{\beta} q_{\alpha}\right) \int \mathcal{D} \alpha_{i} e^{i\left(\alpha_{\bar{q}}+v \alpha_{g}\right) q x} \mathcal{T}_{3}\left(\alpha_{i}\right) \\
& \left.+\frac{1}{q x}\left(q_{\alpha} x_{\beta}-q_{\beta} x_{\alpha}\right)\left(\varepsilon_{\mu} q_{\nu}-\varepsilon_{\nu} q_{\mu}\right) \int \mathcal{D} \alpha_{i} e^{i\left(\alpha_{\bar{q}}+v \alpha_{g}\right) q x} \mathcal{T}_{4}\left(\alpha_{i}\right)\right\}
\end{aligned}
$$

where, $\chi$ is the magnetic susceptibility of the quarks, $\varphi_{\gamma}(u)$ is the leading twist $2, \psi^{v}(u), \psi^{a}(u), \mathcal{A}$ and $\mathcal{V}$ are the twist 3 and $h_{\gamma}(u), \mathbb{A}, \mathcal{T}_{i}(i=1,2,3,4)$ are the twist 4 photon DA's, respectively. The measure $\mathcal{D} \alpha_{i}$ is defined as

$$
\int \mathcal{D} \alpha_{i}=\int_{0}^{1} d \alpha_{\bar{q}} \int_{0}^{1} d \alpha_{q} \int_{0}^{1} d \alpha_{g} \delta\left(1-\alpha_{\bar{q}}-\alpha_{q}-\alpha_{g}\right) .
$$

The explicit expressions of the photon distribution amplitudes (DA's) with different twists are [29]:

$$
\begin{aligned}
\varphi_{\gamma}(u)= & 6 u \bar{u}\left(1+\varphi_{2}(\mu) C_{2}^{\frac{3}{2}}(u-\bar{u})\right) \\
\psi^{v}(u)= & 3\left(3(2 u-1)^{2}-1\right)+\frac{3}{64}\left(15 w_{\gamma}^{V}-5 w_{\gamma}^{A}\right)\left(3-30(2 u-1)^{2}+35(2 u-1)^{4}\right) \\
\psi^{a}(u)= & \left(1-(2 u-1)^{2}\right)\left(5(2 u-1)^{2}-1\right) \frac{5}{2}\left(1+\frac{9}{16} w_{\gamma}^{V}-\frac{3}{16} w_{\gamma}^{A}\right) \\
\mathcal{A}\left(\alpha_{i}\right)= & 360 \alpha_{q} \alpha_{\bar{q}} \alpha_{g}^{2}\left(1+w_{\gamma}^{A} \frac{1}{2}\left(7 \alpha_{g}-3\right)\right) \\
\mathcal{V}\left(\alpha_{i}\right)= & 540 w_{\gamma}^{V}\left(\alpha_{q}-\alpha_{\bar{q}}\right) \alpha_{q} \alpha_{\bar{q}} \alpha_{g}^{2} \\
h_{\gamma}(u)= & -10\left(1+2 \kappa^{+}\right) C_{2}^{\frac{1}{2}}(u-\bar{u}) \\
\mathbb{A}(u)= & 40 u^{2} \bar{u}^{2}\left(3 \kappa-\kappa^{+}+1\right) \\
& +8\left(\zeta_{2}^{+}-3 \zeta_{2}\right)[u \bar{u}(2+13 u \bar{u}) \\
& \left.+2 u^{3}\left(10-15 u+6 u^{2}\right) \ln (u)+2 \bar{u}^{3}\left(10-15 \bar{u}+6 \bar{u}^{2}\right) \ln (\bar{u})\right] \\
\mathcal{T}_{1}\left(\alpha_{i}\right)= & -120\left(3 \zeta_{2}+\zeta_{2}^{+}\right)\left(\alpha_{\bar{q}}-\alpha_{q}\right) \alpha_{\bar{q}} \alpha_{q} \alpha_{g}, \\
\mathcal{T}_{2}\left(\alpha_{i}\right)= & 30 \alpha_{g}^{2}\left(\alpha_{\bar{q}}-\alpha_{q}\right)\left(\left(\kappa-\kappa^{+}\right)+\left(\zeta_{1}-\zeta_{1}^{+}\right)\left(1-2 \alpha_{g}\right)+\zeta_{2}\left(3-4 \alpha_{g}\right)\right)
\end{aligned}
$$




$$
\begin{aligned}
\mathcal{T}_{3}\left(\alpha_{i}\right) & =-120\left(3 \zeta_{2}-\zeta_{2}^{+}\right)\left(\alpha_{\bar{q}}-\alpha_{q}\right) \alpha_{\bar{q}} \alpha_{q} \alpha_{g}, \\
\mathcal{T}_{4}\left(\alpha_{i}\right) & =30 \alpha_{g}^{2}\left(\alpha_{\bar{q}}-\alpha_{q}\right)\left(\left(\kappa+\kappa^{+}\right)+\left(\zeta_{1}+\zeta_{1}^{+}\right)\left(1-2 \alpha_{g}\right)+\zeta_{2}\left(3-4 \alpha_{g}\right)\right) \\
\mathcal{S}\left(\alpha_{i}\right) & =30 \alpha_{g}^{2}\left\{\left(\kappa+\kappa^{+}\right)\left(1-\alpha_{g}\right)+\left(\zeta_{1}+\zeta_{1}^{+}\right)\left(1-\alpha_{g}\right)\left(1-2 \alpha_{g}\right)\right. \\
& \left.+\zeta_{2}\left[3\left(\alpha_{\bar{q}}-\alpha_{q}\right)^{2}-\alpha_{g}\left(1-\alpha_{g}\right)\right]\right\} \\
\tilde{\mathcal{S}}\left(\alpha_{i}\right) & =-30 \alpha_{g}^{2}\left\{\left(\kappa-\kappa^{+}\right)\left(1-\alpha_{g}\right)+\left(\zeta_{1}-\zeta_{1}^{+}\right)\left(1-\alpha_{g}\right)\left(1-2 \alpha_{g}\right)\right. \\
& \left.+\zeta_{2}\left[3\left(\alpha_{\bar{q}}-\alpha_{q}\right)^{2}-\alpha_{g}\left(1-\alpha_{g}\right)\right]\right\} .
\end{aligned}
$$

The constants appearing in the above wave functions are given as [29]:

$\varphi_{2}(1 \mathrm{GeV})=0, w_{\gamma}^{V}=3.8 \pm 1.8, w_{\gamma}^{A}=-2.1 \pm 1.0, \kappa=0.2, \kappa^{+}=0$, $\zeta_{1}=0.4, \zeta_{2}=0.3, \zeta_{1}^{+}=0$ and $\zeta_{2}^{+}=0$.

\section{Appendix B}

In this appendix, we present the explicit expressions for the functions, $F_{1}(0)$, $F_{2}(0), F_{3}(0)$ and $F_{4}(0)$.

$$
\begin{aligned}
F_{1}\left(q^{2}=0\right) & =-\frac{1}{2 m_{\Delta} \lambda_{\Delta}^{2}} e^{\frac{m_{\Delta}^{2}}{M^{2}}}\left(e _ { u } \left\{\langle \overline { d } d \rangle \left[\frac{M^{2}\left[12 E_{1}(x) M^{2}-5 E_{0}(x) m_{0}^{2}\right]}{27 \pi^{2}}\right.\right.\right. \\
& \left.-\frac{2}{81} f_{3 \gamma}\left\{54 E_{0}(x) M^{2}-15 m_{0}^{2}\right\} \psi^{v}\left(u_{0}\right)\right] \\
& +\langle\bar{u} u\rangle\left[\frac{M^{2}\left[3 E_{1}(x) M^{2}\left(8-15 \zeta_{1}\right)-10 E_{0}(x) m_{0}^{2}\right]}{27 \pi^{2}}\right. \\
& \left.\left.-\frac{2}{81} f_{3 \gamma}\left\{54 E_{0}(x) M^{2}-15 m_{0}^{2}\right\} \psi^{v}\left(u_{0}\right)\right]\right\}+e_{d}\left\{\langle\bar{d} d\rangle\left[-\frac{5 E_{1}(x) M^{4} \zeta_{1}}{12 \pi^{2}}\right]\right. \\
& +\langle\bar{u} u\rangle\left[\frac{M^{2}\left[12 E_{1}(x) M^{2}-5 E_{0}(x) m_{0}^{2}\right]}{27 \pi^{2}}\right. \\
& \left.\left.\left.-\frac{2}{81} f_{3 \gamma}\left\{54 E_{0}(x) M^{2}-15 m_{0}^{2}\right\} \psi^{v}\left(u_{0}\right)\right]\right\}\right) \\
F_{2}\left(q^{2}=0\right) & =-\frac{m_{\Delta}}{\lambda_{\Delta}^{2}} e^{\frac{m_{\Delta}^{2}}{M^{2}}}\left(e _ { u } \left\{\langle \overline { d } d \rangle \left[\frac{5 m_{0}^{2}-10 E_{0}(x) M^{2}}{216 \pi^{2}}\right.\right.\right.
\end{aligned}
$$




$$
\begin{aligned}
& \left.+\frac{f_{3 \gamma}}{324 M^{2}}\left\{11 m_{0}^{2}-36 M^{2}\right\} \psi^{a}\left(u_{0}\right)\right] \\
& +\langle\bar{u} u\rangle\left[\frac { 1 } { 2 1 6 \pi ^ { 2 } } \left[5 m_{0}^{2}+6 E_{0}(x) M^{2}\left(-3+2 \eta_{3}+6 \eta_{4}-8 \eta_{5}+2 \eta_{6}-4 \eta_{7}-8 \eta_{8}\right.\right.\right. \\
& \left.-2 \eta_{9}+4 \eta_{10}+24 \zeta_{3}\right)-9 E_{0}(x) M^{2} \mathbb{A}\left(u_{0}+12 E_{1}(x) M^{4} \chi \varphi_{\gamma}\left(u_{0}\right)\right] \\
& \left.\left.+\frac{f_{3 \gamma}}{324 M^{2}}\left\{11 m_{0}^{2}-36 M^{2}\right\} \psi^{a}\left(u_{0}\right)\right]\right\} \\
& +e_{d}\left\{\langle \overline { u } u \rangle \left[\frac{5 m_{0}^{2}-18 E_{0}(x) M^{2}}{216 \pi^{2}}\right.\right. \\
& \left.+\frac{f_{3 \gamma}}{324 M^{2}}\left\{11 m_{0}^{2}-36 M^{2}\right\} \psi^{a}\left(u_{0}\right)\right] \\
& +\langle\bar{d} d\rangle\left[\frac { M ^ { 2 } } { 1 4 4 \pi ^ { 2 } } \left[4 E _ { 0 } ( x ) \left(\eta_{3}+3 \eta_{4}-4 \eta_{5}+\eta_{6}-2 \eta_{7}-4 \eta_{8}\right.\right.\right. \\
& \text { - } \left.\left.\left.\left.\eta_{9}+2 \eta_{10}+12 \zeta_{3}\right)-3 E_{0}(x) M^{2} \mathbb{A}\left(u_{0}+4 E_{1}(x) M^{4} \chi \varphi_{\gamma}\left(u_{0}\right)\right]+\right]\right\}\right) \\
& F_{3}\left(q^{2}=0\right)=-\frac{2 m_{\Delta^{2}}}{\lambda_{\Delta}^{2}} e^{\frac{m_{\Delta}^{2}}{M^{2}}}\left(e _ { u } \left\{\frac{-E_{1}(x) M^{4}\left(u_{0}-1\right) u_{0}}{12 \pi^{4}}\right.\right. \\
& +\frac{f_{3 \gamma} E_{0}(x) M^{2}}{18 \pi^{2}}\left[10 \eta_{1}-8 \eta_{11}-8 \eta_{2}+4 \zeta_{2}\left(1-2 u_{0}\right)-\psi^{a}\left(u_{0}\right)\right] \\
& \left.-\frac{4}{81 M^{4}}\langle\bar{u} u\rangle(\langle\bar{u} u\rangle+\langle\bar{d} d\rangle)\left[-10 m_{0}^{2} \zeta_{3}+6 M^{2}\left(6 \zeta_{3}+3 \xi_{1}+4 \xi_{2}-\xi_{3}\right)\right]\right\} \\
& +e_{d}\left\{\frac{-E_{1}(x) M^{4}\left(u_{0}-1\right) u_{0}}{24 \pi^{4}}\right. \\
& +\frac{f_{3 \gamma} E_{0}(x) M^{2}}{36 \pi^{2}}\left[10 \eta_{1}-8 \eta_{11}-8 \eta_{2}+4 \zeta_{2}\left(1-2 u_{0}\right)-\psi^{a}\left(u_{0}\right)\right] \\
& \left.\left.-\frac{4}{81 M^{4}}\langle\bar{u} u\rangle\langle\bar{d} d\rangle\left[-10 m_{0}^{2} \zeta_{3}+6 M^{2}\left(6 \zeta_{3}+3 \xi_{1}+4 \xi_{2}-\xi_{6}\right)\right]\right\}\right) \\
& F_{4}\left(q^{2}=0\right)=-\frac{4 m_{\Delta^{2}}}{\lambda_{\Delta}^{2}} e^{\frac{m_{\Delta}^{2}}{M^{2}}}\left(e _ { u } \left\{\frac{-E_{1}(x) M^{4}\left(u_{0}-1\right) u_{0}^{2}}{8 \pi^{4}}\right.\right. \\
& +\frac{f_{3 \gamma} E_{0}(x) M^{2}}{18 \pi^{2}}\left[8\left(\eta_{11}+\eta_{2}\right)-12 \zeta_{2} u_{0}^{2}+8 u_{0}\left(\eta_{1}-3 \eta_{11}-\eta_{2}+\zeta_{2}\right)\right.
\end{aligned}
$$




$$
\begin{aligned}
& \left.+\left(u_{0}-2\right) u_{0} \psi^{a}\left(u_{0}\right)\right] \\
& \left.+\frac{4}{81 M^{4}}\langle\bar{u} u\rangle(\langle\bar{u} u\rangle+\langle\bar{d} d\rangle)\left[-10 m_{0}^{2} u_{0} \zeta_{3}+12 M^{2}\left(1+3 u_{0} \zeta_{3}+\xi_{2}-\xi_{3}\right)\right]\right\} \\
& +e_{d}\left\{\frac{-E_{1}(x) M^{4}\left(u_{0}-1\right) u_{0}^{2}}{16 \pi^{4}}\right. \\
& +\frac{f_{3 \gamma} E_{0}(x) M^{2}}{36 \pi^{2}}\left[8\left(\eta_{11}+\eta_{2}\right)-12 u_{0}^{2} \zeta_{2}+8 u_{0}\left(\eta_{1}-3 \eta_{11}-\eta_{2}+\zeta_{2}\right)\right. \\
& \left.+\quad\left(u_{0}-2\right) u_{0} \psi^{a}\left(u_{0}\right)\right] \\
& \left.\left.+\frac{4}{81 M^{4}}\langle\bar{u} u\rangle\langle\bar{d} d\rangle\left[10 m_{0}^{2} u_{0} \zeta_{3}-12 M^{2}\left(3 u_{0} \zeta_{3}+\xi_{2}-\xi_{3}\right)\right]\right\}\right)
\end{aligned}
$$

where, the functions entering the above equations are given as

$$
\begin{aligned}
\eta_{i} & =\int \mathcal{D} \alpha_{i} \int_{0}^{1} d v f_{i}\left(\alpha_{i}\right) \delta\left(\alpha_{q}+v \alpha_{g}-u_{0}\right) \\
\xi_{i} & =\int \mathcal{D} \alpha_{i} \int_{0}^{1} d \bar{v} g_{i}\left(\alpha_{i}\right) \theta\left(\alpha_{q}+v \alpha_{g}-u_{0}\right), \\
\zeta_{i} & =\int_{u_{0}}^{1} d u h_{i}(u) \\
E_{n}(x) & =1-e^{-x} \sum_{k=0}^{n} \frac{x^{k}}{k !}
\end{aligned}
$$

and $f_{1}\left(\alpha_{i}\right)=\mathcal{A}\left(\alpha_{i}\right), f_{2}\left(\alpha_{i}\right)=v \mathcal{A}\left(\alpha_{i}\right), f_{3}\left(\alpha_{i}\right)=\mathcal{S}\left(\alpha_{i}\right), f_{4}\left(\alpha_{i}\right)=\tilde{\mathcal{S}}\left(\alpha_{i}\right)$, $f_{5}\left(\alpha_{i}\right)=v \tilde{\mathcal{S}}\left(\alpha_{i}\right), f_{6}\left(\alpha_{i}\right)=g_{2}\left(\alpha_{i}\right)=\mathcal{T}_{2}\left(\alpha_{i}\right), f_{7}\left(\alpha_{i}\right)=v \mathcal{T}_{2}\left(\alpha_{i}\right), f_{8}\left(\alpha_{i}\right)=$ $v \mathcal{T}_{3}\left(\alpha_{i}\right), f_{9}\left(\alpha_{i}\right)=g_{3}\left(\alpha_{i}\right)=\mathcal{T}_{4}\left(\alpha_{i}\right), f_{10}\left(\alpha_{i}\right)=v \mathcal{T}_{4}\left(\alpha_{i}\right), f_{11}\left(\alpha_{i}\right)=v \mathcal{V}\left(\alpha_{i}\right)$, $g_{1}\left(\alpha_{i}\right)=\mathcal{T}_{1}\left(\alpha_{i}\right), h_{1}(u)=h_{\gamma}(u), h_{2}(u)=\psi^{v}(u)$ and $h_{3}(u)=\left(u-u_{0}\right) h_{\gamma}(u)$ are the photon distribution amplitudes. Note that, in the above equations, $x=s_{0} / M^{2}, \bar{v}=1-v$ and the Borel parameter $M^{2}$ is defined as $M^{2}=\frac{M_{1}^{2} M_{2}^{2}}{M_{1}^{2}+M_{2}^{2}}$ and $u_{0}=\frac{M_{1}^{2}}{M_{1}^{2}+M_{2}^{2}}$. Since the masses of the initial and final baryons are the same, we will set $M_{1}^{2}=M_{2}^{2}$ and $u_{0}=1 / 2$. 